AN OPERATION ON THE STOMACH THAT LOOKS AFTER YOUR HEART

AR Carmichael.DJ Owen,RFGJ King,HM Sue-Ling, D Johnston Academic Unit of Surgery, Leeds General Infirmary, Leeds, UK

Coronary heart disease (CHD) is the commonest cause of death in the western world. The increasing incidence of obesity poses a serious risk of CHD because of its effects on serum lipids, type II diabetes, hypertension and left ventricular hypertrophy. The Framingham group have devised a CHD score based on a 40 year follow-up of over $\mathbf{5 0 0 0}$ men and women. It is robust and validated to predict the risk that CHD will develop in the ensuing 10 years. The Magenstrasse and Mill procedure for morbid obesity involves formation of a lesser curve tube of the stomach connected to normal antral mill and is more physiological than other anti-obesity procedures. We tested the hypothesis the M\&M procedure would provide good protection against the risk of CHD, which is greatly increased in obese people. The Framingham risk score based on patients' age, gender, lipids, cigarette smoking, ECG-LVH, blood pressure and diabetes was used to measure the risk of CHD in 24 obese patients,(23 females) with a median age of 40 years (range 31-48), before and 24 months after the M\&M procedure for morbid obesity. The Framingham score, median (range) was reduced from 9 (3-29) to 3(1-7) with a median percentage reduction of $67 \%(P<0.001)$. Weight was reduced from $137 \mathrm{~kg}$ $(127-200)$ to $91 \mathrm{~kg}(62-114)$ with a median $58 \%$ reduction in excess body weight. Median BMI was reduced from 51(36-61) to $34(27-41) \mathrm{kg} / \mathrm{m}^{2}$. NIDDM was"cured" in all 8 patients while impaired glucose tolerance reverted to normal in a further 3 patients. Five of eight hypertensive patients (SB.P > 160) came off anti-hypertensive medication and their blood pressure reverted to normal. Thus, the M\&M procedure led to a significant reduction both in weight and the risk of CHD in morbidly obese patients.

\section{Audit and inflammatory bowel disease}

PATS FOR PEGS - AUDITED

S White, J Hart, A Torrance, s Clark, P Bottrill and $\mathrm{K}$ Matthewson.

Departments of Gastroenterology, Speech Therapy and Dietetics, Royal Victoria Infirmary, Newcastle Upon Tyne.

Before January 1996 there was no coordinated approach to the management of patients with percutaneous endoscopic gastrostomies (PEG) in this Unit. Medical follow up was by the referring clinician with gastroenterological intervention only when a problem developed. Other disciplines made their individual contribution.

A PBG advice team (PAT), comprising a clinical liaison nurse (CLN), dietitian, speech therapist and gastroenterologist, with back up from a senior dietitian and nurse manager, was established on 1st January 1996. The PAT aimed to provide pre-procedure assessment and counselling, insert the PEG, educate the patient and carers regarding feeding and general care of the PEG, provide ongoing supervision of inpatients, and to proactively review outpatients a minimum of 3 monthly.

In the 5 months before the PAT there were 16 PEG insertions and in the 5 months after there were 22, an increase of $38 \%$. By 18.5 .96 there were 31 patients under the team's care, of which $32 \%$ had motor neurone disease, $26 \%$ had strokes and $10 \%$ had multiple sclerosis. It was planned to review 2 outpatients every two weeks, but demand is such that 2 or 3 are seen each week. The most common problem is leakage around the stoma, a problem easily solved by education of patient and carer. The CIN was expected to devote 1 day/week to PAT duties, but they account for 3 days as she is in demand to provide counselling and advice, and visit outlying institutions when patients are unable to attend hospital.

PEG patients have problems which a proactive PAT is well placed to solve. The CLN fulfills a crucial role in coordinating it.
LONG-TERM OUTCOME OF GASTROSTOMY FEEDING IN DYSPHAGIC STROKE. K Kapur, A James, AB Hawthorne. Dept of Medicine, University Hospital of Wales, Heath Park, Cardiff

Percutaneous Endoscopy Gastrostomy (PEG) is now preferable to nasogastric feeding in dysphagic stroke patients. Short-term studies have shown PEGs are safe and better tolerated. Long-term outcome data is lacking, nor is it clear how early PEG tubes should be placed.

Methods Case notes were obtained for 126 patients who underwent PEG placement for acute dysphagic stroke at University Hospital of Wales and Cardiff Royal Infirmary between 1991 and 1995 . Complete outcome data was available for 120 patients using case notes, PEG follow-up clinic records, GP records and nursing home records. Median follow-up (FU) was 30 months (range 4 - 71).

Results Median age 79 (range 53 - 94). Median interval between stroke and PEG insertion was 22 days, 41 patients had PEG within 2 weeks, 33 in week 3 - 4 and 52 after 4 weeks. $35(28 \%)$ died in hospital, $32(25 \%)$ discharged to long-stay hospital, $42(33 \%)$ to nursing home and $16(13 \%)$ to their own home. Overall $35(28 \%)$ recovered swallow and had PEG removed. 6 (5\%) were taking diet orally but still received some feeding by PEG. 72 (57\%) died with PEG in use (nil oral). 9 (7\%) continued with PEG feeding and NBM at FU. Time

Oral intake, PEG removed

PEG in use, some oral intake

PEG in use, NBM

Died with PEG, NBM

No. available for observation

\begin{tabular}{llllll}
0 & $3 \mathrm{mo}$ & $6 \mathrm{mo}$ & $1 \mathrm{yr}$ & $2 \mathrm{yr}$ & $3 \mathrm{yr}$ \\
\hline & 12 & 19 & 29 & 32 & 33 \\
120 & - & 1 & 1 & 3 & 6 \\
& 65 & 48 & 29 & 10 & 1 \\
& 43 & 51 & 57 & 67 & 71 \\
\hline
\end{tabular}

61 complications occurred in 54 patients. Early: 1 died within $24 \mathrm{hrs,}$ 3 pneumoperitoneum, 1 leak around PEG. Late: aspiration pneumonia 22, site infection 13, tube blocked 12, MRSA 4, fell out 4, snapped 1 . PEGs placed within 14 days of stroke were in use (ie until death or swallow recovery) for $<1$ month in $32 \%$, compared to $20 \%$ and $19 \%$ for PEGs placed either 14-28 days or $>28$ days after stroke $(p=N S)$.

Conclusions Early placement of PEG is worthwhile. Aspiration pneumonia is the commonest complication. Long-term FU is essential as late recovery of swallow ( $>6$ months) occurred in $17 \%$.
AN AUDIT OF THE INVESTIGATION OF 'LONE' IRON DEFICIENCY IN A GASTROINTESTINAL UNIT

SMLaitner, JMT Willoughby, Dept. of Gastroenterology, Lister Hospital, Stevenage, Herts SG1 4AB

Endoscopy records between 1st March 1990 and 28th February 1995 were searched for details of patients investigated for iron deficiency in whom neither the history nor a general examination had offered any pointer to the causative pathology. 128 cases were found to satisfy this criterion.

A diagnosis considered relevant was made in 48 (38\%) patients. Median time from presentation to diagnosis was 1 month (range 0 - 12). A single investigation sufficed in 20 (42\%) of these patients, and only $9(19 \%)$ required more than 2 investigations. The age group with the highest diagnostic rate $(52 \%)$ was that of patients between 65 and 74 . The proportion of the 13 patients diagnosed at age $\mathbf{7 5}$ or over $(30 \%)$ did not differ from that of the 7 patients under $45(33 \%)$, but 6 different gastrointestinal lesions were found in the first of these groups, while in the second there were only 2 pathologies, of which coeliac disease accounted for all except 1 case. As had been expected, the elimination of patients with rectal bleeding skewed the distribution of colonic cancers such that 10 of 16 were right-sided. Less predictable was the finding that only 1 of 12 haemorrhagic lesions in the upper GI tract occurred in a patient under 65.

Upper GI endoscopy was performed in 111 (85\%) patients, 87 times as the first investigation. Its diagnostic yield was $16 \%$, as against $26 \%$ for colonoscopy, $10 \%$ for barium enema, and $7 \%$ for small bowel meal.

A suggested protocol for the investigation of 'lone' iron deficiency, based on data gathered both during the above period and at follow-up, will be presented. 
AN AUDIT OF LONG-TERM PPI PRESCRIBING FOR GORD IN GENERAL PRACTICE.

Clarke F, Hall I, Meekison L, Heading RC. The Health Centre, High Street. Bonnyrigg. Midlothian, UK and University Department of Medicine, Royal Infirmary, Edinburgh, UK.

Allegations about over-prescription of long-term proton pump inhibitor (PPI) therapy for dyspepsia seem to be founded on relatively little data derived from direct review of current prescribing practice. We have obtained data relating to long-term (repeat prescription) PPI therapy from 6 general practices in Lothian in which 38 GP principals are responsible for 57,766 patients. The PPI prescribing patterns of these practices were a reasonable cross-section of the PPI prescribing pattern in the Lothian area.

In the 6 practices, PPI repeat prescriptions were being issued to 0.3$1.6 \%$ of registered patients. The diagnoses warranting treatment were given as gastro-oesophageal reflux disease (GORD) $n=193(52.0 \%$ of total on long-term PPI), peptic ulceration $n=89(24.0 \%)$, unspecified dyspepsia $n=77(20.8 \%)$, duodenitis $n=9(2.4 \%)$, unknown / other $n=3$ $(0.8 \%)$

Amongst the patients with GORD, the possibility of changing their medication to reduce the degree of acid suppression was considered. Available records demonstrated that reduction had previously been attempted unsuccessfully in $55(28.5 \%)$. In a further 3 patients, current medication was evidently inadequate. 7 patients $(3.6 \%)$ had an endoscopic diagnosis of oesophagitis at grade 3 or more. In a further 47 patients $(24.4 \%)$ there had been specific consultant advice to keep the patient on long-term PPI at the current dose. 17 patients (8.8\%) had other major medical conditions which were the more important focus of current attention and clinical management.

These observations indicate that amongst patients receiving long-term PPI therapy for a diagnosis of GORD, only one quarter or thereabouts are obvious candidates for consideration of reduction in their antisecretory therapy.
THE SUCCESS OF AUDITED GUIDELINES IN THE MANAGEMENT OF INFLAMMATORY BOWEL DISEASE

A Read, J de Caestecker, J Cannon, S Longworth, C O'Shea, BJ Rathbone, D Spence, M Stone, ACB Wicks and JF Mayberry.

Gastroenterology, Leicester General Hospital NHS Trust, Gwendolen Road, Leicester LE5 4PW

Consensus guidelines were produced in 1993 by the gastroenterologists, gastro-intestinal surgeons and a cross-section of general practitioners in a single district health authority. The purpose of the guidelines was to develop a seamless pattern of care with a common approach to diagnosis and treatment. It was hoped that they would encourage a movement towards care in the community for many patients and so speed up new consultation rates. The guidelines were professionally published and distributed to all 450 general practitioners in the district. Their introduction was supported by a series of approved (PGEA) meetings.

The value of the guidelines was assessed by an audit of the quality of referral letters, the adherence to guidelines on re-referral and monitoring the outcome of discharges from hospital follow-up.

The results of the study showed that referral letters have not improved since the launch of the guidelines in the amount of information they provide. However, it has showed that only $5 \%$ (3 of 56) of the stable patients who were discharged from out-patients were re-referred with a related problem. These referrals were within the guidelines stated reasons for this. Re-referrals were made between $12-15$ months of their discharge. Guidelines have helped the G.P.s to manage stable patients in the community, and hense the out patient clinics can function more effectively and respond quicker to new or urgent problems.

\section{IS A HOSPITAL PRESCRIBING POLICY FOR}

HELICOBACTER PYLORI ERADICATION COST EFFECTIVE? A Soliman, B Fajemisin, M J Grundman, Depts of Gastroentrology and Pharmacy. Chesterfield \& North Derbyshire Royal Hospital, Chesterfield, Derbyshire.

H Pylori eradication was introduced gradually in this hospital from 1991 without any planning by Medical and Surgical Gastroenterologists.

From July to October 1994 the Pharmacy Department conducted an audit on out-patient prescriptions from the Medical and Surgical Directorates. This revealed a total of 16 different regimes some based on clinical trials some not with community costs varying from $£ 14.00$ to $£ 158.00$ per course with widely differing reported eradication rates

As a result a prescribing policy was introduced from April 1995 with two well established standard regimes: Regime 1 - Omeprazole 20mgs twice daily, Amoxycillin $500 \mathrm{mgs}$ tds and Metronidazole $400 \mathrm{mgs}$ tds all for one week for all patients other than those sensitive to Penicillin who were prescribed Regime 2 - Omeprazole $20 \mathrm{mgs}$ bd, Clarithromycin $250 \mathrm{mgs}$ bd and Tinidazole $500 \mathrm{mgs}$ bd. The respective hospital costs were $£ 8.19$ and $£ 26.02$ per course, community costs $£ 20.33$ and $£ 37.02$ and reported eradication rates $91 \%$ and $96 \%$.

Out-patient prescriptions were re-audited from April to October 1995 Of the 63 prescriptions evaluated $84 \%$ were for the standard regimes: $69 \%$ regime 1 and $15 \%$ regime 2 . Of the remaining $16 \%$ all but $5 \%$ were only marginally different from the agreed regimes.

The July to October prescriptions of 1994 and 1995 were compared identifying hospital savings of $£ 332.00$ (a $42 \%$ reduction in unit costs) and hence anticipated annual savings of $£ 1000.00$ (or $£ 1500.00$ at community prices) with anticipated improvement in eradication.

Thus a prescribing policy can save money and should improve eradication rates.
T144

Asecenemont of Crohn's diceane activity by duplex/colour dopplex eonography. C.Adams, I Britton, C Maguire, R I Russell, E. Leen. Departments of Radiology and Gastroenterology, Royal Infirmary, Glasgow, G31 2ER The assessment of disease activity in patients with Crohn's disease involves the integration of a number of clinical, laboratory and radiological parameters. No single parameter provides a reliable and immediate guide to disease activity. Alm of study. We have investigated the role of Duplex Colour Doppler Sonography (DCDS) of the superior mesenteric artery (SMA) as a non-invasive and immediate reflection of activity of Crohn's disease. Mothode and Pationts. Baseline SMA volume flow rates, the response to a food challenge and peak flow rates were recorded in 12 controls, 18 patients with active, and 21 in patients with inactive, crohn's disease. Some patients with active disease were followed longitudinally to monitor their responses following the introduction of systemic steroids. Subjects were studied supine after an 8 hour fast. Baseline flow rates were recorded initially and then a standard food challenge was given (150ml Ensure Plus). Repeat flow rates were recorded every $5 \mathrm{~min}$ for $10 \mathrm{~min}$ and then every 10 min for up to $60 \mathrm{~min}$. Reault. Baseline flow rates were not significant in differentiating active from inactive disease. The time interval from food challenge to peak SMA flow rate was significantly higher in patients with inactive disease compared to patients with untreated active disease $(33.2 \mathrm{~min}+$ 2.64 (SE) v $17.64 \mathrm{~min}+2.13 \mathrm{p}<0.001$ ). A similar, though less significant, difference was noticed with controls v. untreated active patients and with treated $v$ untreated active patients (both $\mathrm{p}<0.05$ ). Those patients followed up after the introduction of systemic steroids showed a significant upward trend in their time to peak flow $(23.12 \mathrm{~min} .+2.06 \mathrm{v} 33.6$ $+2.27 ; p<0.003$. Concluelon. This technique is useful in of fering an immediate, non invasive means of assessing Crohn's disease activity. Further longitudinal follow up data is important. 
ANCA AND HLA GENES IN NORTH EUROPEAN PATIENTS WITH CROHN'S DISEASE

Jack Satsangi ${ }^{1}$, Carol Landers ${ }^{2}$, Ken I Welsh ${ }^{3}$, Stephan Targan ${ }^{2}$, Derek P Jewell ${ }^{1}$. Nuffield Departments of Medicine ${ }^{1}$ and Surgery ${ }^{3}$, Oxford Radcliffe Hospitals, Oxford, UK and the Inflammatory Bowel Disease Center ${ }^{2}$, Cedars Sinai Medical Center, Los Angeles, USA.

Background Our recent data provide evidence that HLA genes may determine ANCA status in ulcerative colitis (UC): in North European patients, ANCA prevalence and ELISA binding was increased in DR3 DQ2 TNF-2-positive UC patients, whereas in California, ANCA status was associated with HLA DR2. Aim To examine the relationship between ANCA status, HLA genotype and clinical patterns of Crohn's disease. Patients 116 North European patients with Crohn's disease. All were UK residents. Details of age of onset, extent, family history and surgery were available. Controls 58 healthy ethnically matched hospital workers. Methods ANCA typing was performed using a fixed neutrophil ELISA and incirect immunofluorescence on ELISA positive (binding $>10 \%$ of positive controls) samples. Genotyping for HLA DRB1, DQB1 and for the bi-allelic polymorphism at residue -308 of the TNF promoter region was performed using sequence specific primers. Results $46 \%$ of CD patients $(18.9 \%$ pANCA, $27.1 \%$ cANCA and $29.3 \%$ of healthy controls $(12.1 \%$ pANCA, $15.5 \%$ CANCA) were ANCA positive. ELISA binding in CD was low compared with UC (mean \pm SD $14.5 \pm 18.8$ v $40.5 \pm$ 44.1). pANCA-positive $C D$ samples displayed higher binding than cANCA positive $C D$ (mean $\pm S D 32.8 \% \pm 34.4 \%$ versus $15.7 \% \pm$ $7.0, p=0.028$ ). Only $15 \overline{C D}$ samples $(13 \%)$ attained ELIS $\bar{A}$ binding greater than $20 \%$ - all of these patients had colonic disease. The prevalence of ANCA (59.1\% versus $39.4 \%, \mathrm{p}=0.04$ ) and ELISA binding $(20.2 \% \pm 28 \%$ versus $8 \% \pm 11.1 \%, \mathrm{p}=0.01)$ were increased in DQ7 positive patients, compared with DQ7 negative patients. Conclusions Low concentrations of ANCA are detectable in plasma of many $C D$ patients and healthy subjects. High concentrations of ANCA are restricted to a proportion of CD patients with colonic involvement, and may represent a serological marker of disease heterogeneity.

ORO-FACIAL GRANULOMATOSIS AND CROHN'S DISEASE : RESULTS OF ILEO-COLONOSCOPY. Sanderson JD, Barnard KM, Lucas S, Challacombe SJ. Departments of Oral Medicine, Histopathology and Gastroenterology, Guy's Hospital, London SE19RT.

Oro-facial granulomatosis (OFG) defines a group of granulomatous conditions which include oral Crohn's disease, Melkersson-Rosenthal syndrome, cheilitis granulomatosa and oral sarcoidosis. OFG involves typically one or more of the lip, buccal mucosa, floor of mouth and gingiva. Most patients with OFG do not have gut symptoms and the proportion of OFG patients with gut inflammation has not been previously well defined.

We have studied a series of 21 cases of OFG. 16/21 (76\%) had more than 4 oral sites involved, 18/21 (86\%) had lip involvement and the median age of onset of oral disease was 19 years. Colonoscopy has been performed in 10 cases with no gut symptoms. Abnormalities were detected in the ileum or colon in $8 / 10$ (80\%) cases. Macroscopic abnormality was present in only 2 cases (ileal apthae $(n=1)$, ileal erythema $(n=1))$. Microscopic inflammation was detected in a further 6 cases. Abnormalities were found on ileoscopy alone in $3 / 10$ cases (30\%). Ileal and/or colonic granulomas were detected in $7 / 10$ cases (70\%). OFG patients with gut inflammation had a lower median age of onset than those without (19.5yrs vs. 54yrs.).

Ileocolonoscopy detects previously undiagnosed gut inflammation in a significant number of patients presenting with OFG, especially those presenting at a younger age. The significance and natural history of gut inflammation in OFG needs to be established in further studies. The results of this provisional study suggest that OFG may be a manifestation of Crohn's disease in a majority of cases.
T147

\section{IMHIBITION BY HEPARIN OF NEUTROPHIL ADHESION TO HUMAN}

\section{IWTESTIMAL MICROVASCULAR EMDOTHELIAL CELLS IIV VITRO.}

J Wilson, SL Bloom and JM Rhodes. Department of Medicine, University of Liverpool, Liverpool, L69 3BX, UK.

Background. There have been several reports of clinical improvement and histological evidence of remission associated with use of heparin in patients with Ulcerative colitis (UC). Patients with UC have extensive infiltration of neutrophils into the lamina propria and as adhesion of leucocytes to the gut endothelium is a central event in the inflammatory process, we have used isolated human intestinal microvascular endothelial cells (HIMECs) to examine the effects of heparin on the adhesion of neutrophils in vitro.

Mothods. HIMECs were isolated from specimens of bowel (colon and small intestine) obtained from patients undergoing resection for colonic cancer or inflammatory bowel disease as previously described (Gut 38: A635, 1996), and cultured in endothelial growth medium (Clonetics) containing 20\% pooled human $A B$ serum. Neutrophils were derived from fresh peripheral venous blood using MonoPoly resolving medium (ICN) and labelled with ${ }^{51}$ Chromium for 1 hour. Endothelial monolayers grown to confluence in $\mathbf{2 4}$ well plates were treated with various concentrations of either high molecular weight heparin (Monoparin) or low molecular weight heparin (Fragmin) for up to six hours, and then overlaid with $5 \times 10^{5}$ neutrophils/well. After 30 minutes, neutrophil adhesion was assessed by scintillation counting and expressed as counts/well.

Results. Untreated endothelial monolayers bound $5 \%$ of the neutrophils added, however, pre-treatment of endothelial monolayers with both high and low molecular weight heparin significantly blocked the adherence of neutrophils by up to $50 \%$ ( $n-3 ; * p<0.05$ ANOVA)

\begin{tabular}{lcccc}
\multicolumn{5}{c}{ Heparin Concentration (Units/ml) } \\
High M.W. & Untreated & 0.1 & 1.0 & 10 \\
Low M.W. & $190 \pm 3.7$ & $92 \pm 10^{*}$ & $119 \pm 2.7^{*}$ & $124 \pm 5.7^{*}$ \\
\hline & $184 \pm 12.4$ & $105 \pm 15.9^{*}$ & $102 \pm 16.4^{*}$ & $120 \pm 5.1^{*}$
\end{tabular}

Conclusions. The pre-treatment of HIMECs with both heparins inhibits the binding of neutrophils indicating that they disrupt the adhesion mechanisms involved in leucocyte-endothelial interactions. This suggests that low molecular weight heparin may have therapeutic potential in the treatment of Inflammatory Bowel Disease.

MUCOSAL REGENERATION IS ASSOCIATED WITH A DECLINE OF EPITHELIAL DEFENCE MECHANISMS. HRH Patel, FC Campbell, Dept of Surgery, Ninewells Medical School, Dundee.

Intestinal diseases associated with recurrent mucosal regeneration eg inflammatory bowel disease, have increased cancer incidence. We propose the hypothesis that undifferentiated progenitor epithelium which predominates during early regeneration, may lack specific functional defences and thus provide a window of susceptibility to genotoxic injury. This study investigates phase I and II detoxification isoenzymes relevant to genotoxic injury and tissue maturation, using a novel model of mucosal regeneration.

Methods: Disaggregated intestinal progenitor epithelium was grafted subcutaneously and selectively recovered at different temporal stages of regeneration. Expression of cytochrome P450 mono-oxygenase (CYP P450 isoforms 1A1,1A2,2B1, 2B2,2C6, 3A1,4A1) and glutathione-S-transferase (GST; Ya,Yc,Yk,Yb1 Yf) were sought by immunoblot at different temporal stages (3, $7,10,14$ and 21 days) after grafting. CYP P450 and GST activity were assessed using specific substrates (eg, ethoxyresorufin [CYP1A], 1-chloro-2,4-dinitrobenzene (CDNB) [GST]). Results were compared against age matched control rat small intestine. Studies were carried out on regenerating vs intact tissue from 8 intestines, on 3 occasions.

Results: Component processes of the regeneration response were identified viz epithelial colony formation, growth, cytodifferentiation and neomucosal morphogenesis. CYP P450, CYP P450 2B1,2B2,2C6 and GST Ya protein expression and activity were decreased or absent in regenerating intestine compared to control.

Conclusions: Phase I and II detoxification isoenzyme expression and activity appear reduced during early mucosal regeneration. These findings may have relevance to genotoxic injury and carcinogenesis associated with regeneration. 